\title{
Why Did I Choose Pathology as a Career?
}

Robert Ta'

About the Author: Robert graduated from medical school from the University of Dublin in Dublin, Ireland. He will be starting his PGY-1 residency program in anatomical and clinical pathology at the Beth Israel Deaconess Medical Center / Harvard Medical school in Boston.
Editor: Omar Aboshady. Submission: 2017-01-04 Acceptance: $2017-05-06$

\section{The Experience}

It seems that the majority of individuals stumble upon the field of pathology by chance. I was no exception to this rule. Since entering medical school, I thought that I was destined to become a surgeon. Fortunately, during my clinical clerkship years, the pathologists at our medical school had a major influence on the medical curriculum. There was to be a mandatory pathology rotation for four weeks and there was no escape. However, this experience generated the spark for my excitement to be entering into the field of pathology.

My first encounter with the field of pathology actually began a long time ago when I was doing research during my graduate studies. We had completed a seven month long project to try and detect a fluorescent compound crossing the blood brain barrier into the brain of a mouse. However, the imaging technique did not work and we were about to scrap the whole experiment. After several discussions, we concluded that histology would be the best way to salvage the work. I spent months analyzing histological slides of mouse brains to determine if the fluorescent agent made it in to where we wanted it to go. It worked and we were able to prove the function of the contrast agent using basic histology!

During medical school, my first exposure to pathology was in the second year pathology lectures. However, that was not the turning point for me to decide to enter the field. In the next year, I was randomly selected to undergo the mandatory pathology rotation. There was exposure to anatomical pathology, autopsy, clinical chemistry, microbiology, and molecular pathology. During anatomical pathology, we would observe resident's gross specimens and have our own set of unknown slides to go through. Autopsies were very involved and also interesting to work out what was the cause of death. Clinical chemistry was stimulating as the rotation allowed us to work up metabolic abnormalities and watch how residents dealt with inquiries from local family physicians and doctors in the main hospital. Microbiology and molecular pathology were much more laboratory based but it was a very scientific experience, for example, on how to logically reason out how to decipher which bacteria was to be tested based upon the clinical history.

After this rotation, I finally got a chance to observe what pathologists do on a daily basis and it completely changed my perspective. Although most pathologists do not deal directly with patients they have plenty of interpersonal interactions with laboratory staff, trainees, referring physicians, and medical students. I had the opportunity to be present at multi-disciplinary team meetings or tumour boards and the pathologist presents the diagnosis and describes the microscopic and macroscopic findings, which all directly drive patient care. It was at this moment that I realized how valuable information from pathologists were in helping other physicians determine what the next steps are in patient management. In some cases, the diagnosis given by pathologists will also dictate the potential treatment options available for patients.

After this enlightening experience, I decided to follow up and apply for electives in pathology. I did two weeks of anatomical pathology in Canada and a further four weeks in the United States. I had an opportunity to work with some world-class pathologists and I knew that this field was for me. I had a chance to work with some of the latest technologies such as laser micro-dissection, where I was acting as a pseudo-surgeon cutting out tumour cells from a several micron thick tissue section using a laser under the microscope.

However, not all of my experiences in choosing a career in pathology were all positive. After deciding and discussing with faculty, clinicians, residents, and medical students, most were completely surprised at my answer to "what do you want to specialize in after you graduate?" I received demeaning looks and given unhelpful comments such as "you must really hate dealing with people... have no clinical skills... no social skills... must be only interested in research". However, the most common remark was "you must love working with dead people". Every so often, I encountered compliments in disguise such as "but you're great with patients, I don't understand why you would want to go into pathology?" I understand that there is a particular type of stigma attached to this field but I take all of these various comments with a grain of salt. Deep down, I know that my work as a future pathologist will be making a difference in many living patients (unless I truly do decide to go into forensic pathology). I was extremely fortunate to meet several surgeons along my path who did truly understand the value of a pathologist and supported my decision to enter this field.

For comparison, in the 2017 National Resident Matching

School of Medicine, Trinity College Dublin, University of Dublin 
Program (NRMP), there were 1281 post-graduate year 1 (PGY1) positions available for categorical surgery while 601 spots for pathology (National Resident Matching Program. Advance Data Tables: 2017 Main Residency Match, 2017. Available from: http://www.nrmp.org/wp-content/uploads/2017/03/AdvanceData-Tables-2017.pdf. cited 2017 April 16). However, only $35.9 \%$ of US graduates decided to enter pathology while $78.5 \%$ of US graduates matched to a general surgery program. There was a $2.1 \%$ decrease in the number of local graduates matching to a pathology residency program this year compared to 2016 . However, this may have been explained by the decrease in number of US applicants and a subsequent increase in the number of PGY-1 pathology residency positions compared to last year's match results. In addition, medical schools do not actively promote pathology as a specialty career and many medical students will have never rotated through a pathology department. Thus, stereotypes about pathology come from within the profession such as: "pathologists didn't like people", "they had poor communication skills" or "they spent all day with dead bodies" (Schubert M. The last Respite of the Socially Inept? The Pathologist, 2017. Available from: https:// thepathologist.com/issues/the-last-respite-of-the-sociallyinept/the-last-respite-of-the-socially-inept/. cited 2017 April 16). Therefore, the overwhelming majority will unfortunately base their entire knowledge of the field upon negative stereotypes from outside and inside medicine.

However, I believe that the future of pathology is extremely bright. Laboratory testing with next generation sequencing allows us to scrutinize each patient's genome. I envision that pathologists will be able to give a diagnostic report to a clinician, for example a family physician, detailing the potential lifetime risks for cancers to guide the history and physical exam.1 This information can help determine what drug is most efficient based on the enzymatic makeup of a patient to determine which pharmacological therapies would be the most beneficial. Finally, we now know that every tumour is different and by analyzing the genetic makeup of a tumour we can determine whether it will respond to a particular chemotherapy.1 Although the term "personalized medicine" has been around for some time, it is only now that we can see the power of laboratory testing playing a more significant role in clinical medicine. The prospect of all this scientific and technological development makes me extremely excited to be entering this field. Even if you are determined to become a surgeon (like I was), I encourage all medical students to rotate through your local friendly pathology department to learn the importance of what we do and how we can help you take care of your patients better.

\section{References}

1. Tonellato PJ, Crawford JM, Boguski MS, Saffitz JE. A national agenda for the future of pathology in personalized medicine: report of the proceedings of a meeting at the Banbury Conference Center on genome-era pathology, precision diagnostics, and preemptive care: a stakeholder summit. Am J Clin Pathol. 2011;135(5):668-72.

2. Collins FS, Varmus H. A New Initiative on Precision Medicine. NEJM. 2015;372(9):793-5.

\footnotetext{
Acknowledgments

The author would like to thank all those individuals who always supported him in whatever he decided to pursue.

Conflict of Interest Statement it Funding

The author has no conflict of interest to disclose.

Author Contributions

Conception and design the work/idea, write the manuscript, approval of the final version: RT.

Cite as:

Ta R. Why Did I Choose Pathology as a Career? Int J Med Students. 2017 Jan-Apr;5(1):61-62.
} 